\title{
Ação da Chuva sobre a EficiênCia de Glyphosate no Controle de Eichhornia crassipes E Pistia stratiotes ${ }^{1}$
}

\author{
Action of Rain under the Effectiveness of Glyphosate in Controlling Eichhornia crassipes and \\ Pistia stratiotes
}

\author{
SOUZA, G.S.F. ${ }^{2}$, CAMPOS, C.F. ${ }^{3}$, MARTINS, D. ${ }^{4}$ e PEREIRA, M.R.R. ${ }^{5}$
}

\begin{abstract}
RESUMO - Com o objetivo de avaliar o efeito da chuva ocorrida em diferentes períodos de tempo após a aplicação do herbicida glyphosate, foram conduzidos dois estudos, sendo um com Eichhornia crassipes e outro com Pistia stratiotes. Ambos os experimentos foram instalados no delineamento inteiramente casualizado, com quatro repetições. Foi simulada uma chuva de $12 \mathrm{~mm}$ durante cinco minutos em diferentes periodos de tempo (Oh, 2h, 4h, 6h, 12h, 24h e não simulação de chuva) após a aplicação de glyphosate, na dose de 2.160 g e.a. ha-1 (4,5 L p.c. ha ${ }^{-1}$ da formulação Rodeo) $+0,5 \%$ v/v de Aterbane. Foram realizadas avaliações visuais de controle das plantas daninhas aos 7, 14, 21 e 28 dias após a aplicação (DAA). O aumento do tempo sem a ocorrência de precipitações após a aplicação de glyphosate proporcionou maior eficiência no controle das duas espécies; o tratamento sem ocorrência de chuva e os com ocorrência a partir de duas horas da aplicação de glyphosate controlaram de forma visual as plantas de E. crassipes após 14 DAA e de $P$. stratiotes somente a partir de 21 DAA. O tratamento com ocorrência de chuva imediatamente após a aplicação do herbicida mostrou controle satisfatório das duas espécies de plantas daninhas aquáticas somente aos 28 DAA.
\end{abstract}

Palavras-chave: aguapé, alface-d'água, herbicida, planta aquática, planta daninha.

\begin{abstract}
Two experiments were carried out to evaluate the effect of rain at different periods of time after glyphosate application, one with Eichhornia crassipes and the other with Pistia stratiotes, both arranged in a randomized design with four replications. The treatments consisted of simulating a $12 \mathrm{~mm}$ rainfall for five minutes, at different periods of time $10 \mathrm{~h}, 2 \mathrm{~h}, 4 \mathrm{~h}, 6 \mathrm{~h}, 12 \mathrm{~h}, 24 \mathrm{~h}$, and no rain simulation) after glyphosate application at a dose of 2, $160 \mathrm{~g}$ a.e. ha $\mathrm{a}^{-1}\left(4.5 \mathrm{~L} \mathrm{c.p}\right.$. ha $\mathrm{a}^{-1}-$ Rodeo formulation) $+0.5 \% \mathrm{v} / v$ aterbane. Visual evaluations of weed control at 7, 14, 21, and 28 days after application (DAA) were conducted. The increase in time without rainfall after glyphosate application provided an excellent control for the two species studied and the treatment without rainfall. The occurrence of rainfall after two hours of glyphosate application effectively controlled $\boldsymbol{E}$. crassipes plants after 14 DAA and $\boldsymbol{P}$. stratiotes after only 21 DAA. Treatments with rainfall immediately after herbicide application showed a good control for the two species of aquatic weeds only at $28 D A A$.
\end{abstract}

Keywords: hyacinth plant, water lettuce, herbicide, aquatic plant, weed.

\section{INTRODUÇÃO}

Nos sistemas aquáticos, em razão da poluição, das alterações dos niveis dos lagos e represas, ou por outras atividades promovidas pelo homem, pode ser observado o efeito do desequilíbrio, externado pela presença de plantas daninhas aquáticas que até então passavam despercebidas ou não se mostravam problemáticas (Foloni \& Pitelli, 2005).

Recebido para publicação em 14.1.2011 e na forma revisada em 18.2.2011.

2 Mestrando, Faculdade de Ciências Agronômicas, Universidade Estadual Paulista “Júlio de Mesquita Filho" - FCA/UNESP, Caixa Postal 237, 18610-307 Botucatu-SP, <guilhermesasso@fca.unesp.br>, ${ }^{3}$ Graduando de Agronomia, FCA/UNESP, Botucatu-SP; ${ }^{4}$ Professor Livre Docente, Dep. de Produção Vegetal, FCA-UNESP, Botucatu-SP, <dmartins@fca.unesp.br>; ${ }^{5}$ Doutorando, FCA/UNESP, Botucatu-SP. 
Em algumas condições tróficas, populações específicas de macrófitas são mais favorecidas que as demais espécies existentes e acabam por desenvolver densas e extensas colonizações, com expressivos efeitos sobre a dinâmica biológica do sistema, bem como sobre os múltiplos usos do corpo hídrico, podendo provocar vários problemas, como: acúmulo de lixo e outros sedimentos, proliferação de vetores de doença, dificuldades na navegação e prejuízos ao turismo e à pesca; nesses casos, podem ser denominadas de daninhas. Essas plantas afetam o transporte de cargas das hidrovias, dificultando as rotas de navegação, além dos prejuízos às usinas hidrelétricas na geração de energia e estações de tratamento de resíduos, o que incrementa os custos de manutenção (Tanaka et al., 2002; Marcondes et al., 2003; Daniel et al., 2006).

As plantas daninhas aquáticas flutuantes são as que causam os mais sérios e difundidos problemas em nível mundial, com comunidades cobrindo extensas áreas, levando a uma série de problemas, como: redução da penetração de luz e da capacidade fotossintética dos vegetais imersos; elevação dos indices de evaporação da área infestada; dificuldade de navegação; danos aos equipamentos de irrigação; prejuízo do desempenho de usinas hidroelétricas; e, até mesmo, abrigo de animais indesejados e vetores de doenças (Kissmann, 1997).

De acordo com Branco (1986), existem várias espécies de macrófitas com potencial para causar prejuízos em ecossistemas aquáticos. Diversos levantamentos de infestação de plantas aquáticas realizados no Brasil, como os de Martins et al. (2008), Pitelli et al. (2008) e Martins et al. (2009a), mostraram que, entre as cinco espécies mais encontradas nesses levantamentos, realizados nos anos de 1998, 2003 e 2002, respectivamente, estão presentes plantas de alface-d'água (Pistia stratiotes) e aguapé (Eichhornia crassipes).

O aguapé caracteriza-se por ser uma planta aquática flutuante livre, nativa da América do Sul, pertencente à família Pontederidaceae; reproduz-se sexuadamente por sementes, as quais são viáveis por pelo menos 15 anos no sedimento dos corpos d'água (Holm \& Yeo, 1980), e de forma assexuada por estolões. Essa espécie é uma das plantas daninhas mais agressivas do mundo e tem sido motivo de grande preocupação nas represas da companhia Light, já que plantas dessa espécie tendem a cobrir a superficie da água, com perigo de serem absorvidas ou entupirem as grades de proteção dos sistemas de turbinas geradoras de energia elétrica. No reservatório de Americana, a área total infestada com plantas aquáticas era de 191 ha em junho de 2001 e havia crescimento médio mensal de 2,27\% (Velini et al., 2002; Swaine et al., 2006).

A alface-d'água é também nativa do continente sul-americano, pertencente à família Araceae; rapidamente, foi levada para vários locais do mundo, em decorrência do caráter ornamental de sua folhagem, podendo ser encontrada em ambientes naturais em todas as regiões de clima não muito frio (Cícero et al., 2007). É uma macrófita aquática flutuante livre, com distribuição cosmopolita tropical, e nos locais onde foi introduzida causa inúmeros problemas aos usos múltiplos dos corpos hídricos (Kissmann, 1997). Alterações antropogênicas, como a eutrofização e o aquecimento global, podem promover o crescimento de $P$. stratiotes e provocar a expansão de sua área de distribuição (Cancian et al., 2009).

Entre os possiveis manejos dessas plantas, com a finalidade de reduzir o seu desenvolvimento ou mesmo de eliminá-las, o controle químico tem-se mostrado a opção mais eficaz e viável no controle de plantas aquáticas (Martins et al., 2009b). O herbicida glyphosate é um inibidor da EPSPS, com ação sistêmica, facilmente translocável e provoca a morte do sistema radicular e de estruturas reprodutivas de plantas perenes, como bulbos e tubérculos, com grande potencial de uso em ambientes aquáticos (Kruse et al., 2000).

Deuber (1982) cita estudos mostrando que a absorção dos herbicidas é limitada pela quantidade que atravessa a cutícula da folha, e esta é influenciada pelas condições ambientais, pela espécie daninha e pelas características dos herbicidas. No entanto, após atingir a superficie foliar, o herbicida está sujeito a vários destinos: escorrer, ser lavado pela ocorrência de chuva, secar e formar substância amorfa, cristalizar após a evaporação do solvente ou, ainda, penetrar na cutícula e permanecer retido nela, não sendo translocado (Werlang et al., 2003). A consequência 
imediata disso é a menor absorção e, consequentemente, menor eficiência do herbicida.

O período crítico entre aplicação do herbicida em pós-emergência e ocorrência de chuva varia de acordo com o tipo de formulação, com a dose empregada, com a solubilidade do produto na água, com as espécies de plantas daninhas, com as condições de desenvolvimento destas e com a quantidade de chuva (Anderson \& Arnold, 1984; Pires, 2000).

Diversos estudos, como os de Jakelaitis et al. (2001), Pedrinho Júnior et al. (2002), Martini et al. (2003), Werlang et al. (2003) e Monquero \& Silva (2007), avaliam a eficiência do herbicida glyphosate submetido à ocorrência de chuvas após sua aplicação no controle de diversas plantas daninhas terrestres; contudo, em se tratando do efeito da chuva na eficiência de glyphosate no controle de plantas daninhas aquáticas, são escassos os trabalhos realizados e publicados na literatura. Portanto, o presente trabalho teve como objetivo avaliar o efeito da chuva, ocorrida em diferentes períodos de tempo, na ação de herbicida glyphosate no controle de $E$. crassipes e $P$. stratiotes.

\section{MATERIAL E MÉTODOS}

Dois estudos foram conduzidos em casa de vegetação, sendo um com plantas da espécie $P$. stratiotes e outro com E. crassipes, as quais foram mantidas em vasos plásticos de $15 \times 15 \times 15 \mathrm{~cm}, \operatorname{com} 2,5$ litros de água, sendo utilizada apenas uma planta por vaso para as duas espécies.

Ambos os experimentos foram instalados no delineamento inteiramente casualizado, com quatro repetições; os tratamentos estudados constaram da simulação de uma chuva de $12 \mathrm{~mm}$, durante cinco minutos, em diferentes períodos de tempo ( $0 \mathrm{~h}, 2 \mathrm{~h}, 4 \mathrm{~h}, 6 \mathrm{~h}, 12 \mathrm{~h}, 24 \mathrm{~h}$ e não simulação de chuva) após a aplicação de glyphosate, na formulação Roundup Rodeo, a 2.160 g e.a. ha ${ }^{-1}\left(4,5\right.$ L p.c. ha $\left.{ }^{-1}\right)+0,5 \%$ v/v de Aterbane.

A simulação da chuva foi realizada sob um sistema estacionário de aplicação de água, regulado para a aplicação da chuva pretendida. Ressalta-se que o período de $\mathrm{Oh}$ para a simulação da chuva ocorreu imediatamente após a pulverização do glyphosate (não mais do que trinta segundos), sendo esse o tempo necessário entre o término da pulverização do glyphosate e a partida do sistema estacionário de aplicação da chuva.

A aplicação do herbicida foi feita através de um pulverizador costal, com pressão constante de $\mathrm{CO}_{2}$ e consumo de calda de $200 \mathrm{~L} \mathrm{ha}^{-1}$. A barra de aplicação estava equipada com duas pontas tipo jato plano Teejet XR 11002VS, distanciadas $50 \mathrm{~cm}$ entre si.

O controle das plantas foi avaliado visualmente aos 7, 14, 21 e 28 dias após a aplicação (DAA), por meio de uma escala percentual de notas, em que zero representava nenhum controle e $100 \%$ o controle total das plantas (SBPD, 1995). Os parâmetros utilizados para o estabelecimento das notas visuais de controle foram: paralisação do crescimento, quantidade e uniformidade das injúrias e capacidade de rebrota das plantas.

Os resultados foram submetidos à análise de variância pelo teste $\mathrm{F}$, sendo as médias dos tratamentos comparadas pelo teste de Tukey $(\mathrm{p}<0,05)$.

\section{RESULTADOS E DISCUSSÃO}

No primeiro estudo, em que se avaliou a ocorrência de chuva após a aplicação de glyphosate no controle das plantas de E. crassipes, observa-se que, aos 7 DAA, as plantas de aguapé não foram controladas de forma eficaz por nenhum dos tratamentos estudados, independentemente do período de chuva simulado após a pulverização do herbicida, porém há maior fitointoxicação das plantas no tratamento sem a ocorrência de chuva (Figura 1).

Já aos 14 DAA, verifica-se que os tratamentos com períodos de ocorrência de chuva a partir de duas horas após a aplicação do herbicida e quando da não ocorrência de chuvas apresentaram médias de controle da planta daninha consideradas de boas a excelentes, sempre acima de $84 \%$ (Figura 2), corroborando Neves et al. (2002), que, ao avaliarem a eficiência de vários herbicidas no controle de E. crassipes, entre estes o glyphosate, também observaram excelentes 


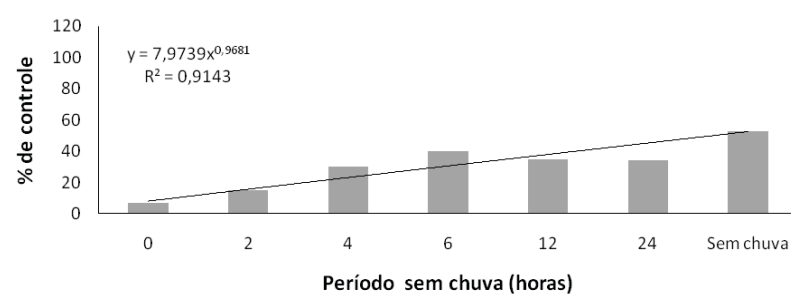

Figura 1 - Porcentagem de controle de plantas de Eichhornia crassipes aos 7 dias após a aplicação do herbicida glyphosate $(2.160$ g e.a. ha-1) $+0,5 \%$ v/v Aterbane, submetidas a diferentes intervalos sem chuva. Botucatu-SP, 2009.

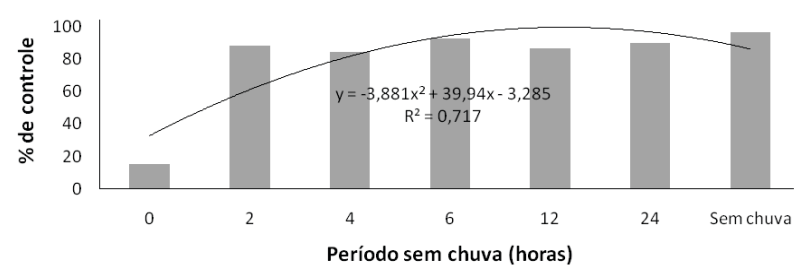

Figura 2 - Porcentagem de controle de plantas de Eichhornia crassipes aos 14 dias após a aplicação do herbicida glyphosate $(2.160$ g e.a. ha-1 $)+0,5 \%$ v/v Aterbane, submetidas a diferentes intervalos sem chuva. Botucatu-SP, 2009.

médias de controle com simulação de chuvas a partir de três horas da aplicação do herbicida, já aos 10 DAA. Esse fato não se verificou com ocorrência de chuva logo após a aplicação do herbicida, o que reduziu a eficiência do controle do glyphosate em mais de 69\%.

Na avaliação realizada aos 21 DAA, notase que todos os tratamentos com ocorrência de chuva a partir de duas horas da aplicação do herbicida mostraram elevadas médias de controle das plantas de aguapé, atingindo o controle máximo da planta daninha na maioria dos tratamentos. O tratamento com precipitação logo após a aplicação do herbicida continuou a influenciar negativamente o seu efeito fitotóxico sobre essa espécie, reduzindo a média de controle do glyphosate em até $72,5 \%$, quando comparado com o tratamento sem ocorrência de chuva (Figura 3).

Na última avaliação, aos 28 DAA, todos os tratamentos com intervalo de tempo para a ocorrência de chuva após a aplicação do herbicida glyphosate maior do que duas horas apresentaram controle total das plantas de aguapé (Figura 4). Já o menor intervalo de tempo estudado, trinta segundos, reduziu a eficiência de controle do herbicida, apresentando média de controle de apenas $73,75 \%$.
Mesmo sendo estatisticamente igual à dos tratamentos, biologicamente, essa média de controle não representa um percentual aceitável para essa espécie. Martins et al. (2002), utilizando glyphosate no controle de aguapé sem posterior simulação de chuva, observaram controle de $100 \%$ da planta daninha já aos 20 DAA, o que reforça o fato de que a ocorrência de chuvas a partir de duas horas da aplicação de glyphosate, na formulação Roundup Rodeo, não afeta sua eficiência de controle de plantas de E. crassipes.

No segundo estudo, realizado com plantas de $P$. stratiotes, foi possivel observar que aos 7 DAA dos tratamentos químicos, com exceção do tratamento que recebeu chuva logo após a aplicação do herbicida, todos os demais proporcionaram algum efeito fitotóxico às plantas de alface-d'água (Figura 5). Verifica-se que os tratamentos que proporcionaram melhor controle da planta daninha foram o que recebeu chuva após 24 horas da aplicação do herbicida e o que não recebeu chuva, mas as suas médias de controle não foram ainda satisfatórias.

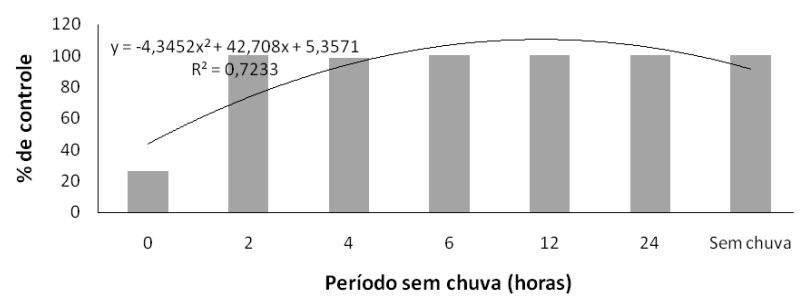

Figura 3 - Porcentagem de controle de plantas de Eichhornia crassipes aos 21 dias após a aplicação do herbicida glyphosate $\left(2.160\right.$ g e.a. ha $\left.{ }^{-1}\right)+0,5 \%$ v/v Aterbane, submetidas a diferentes intervalos sem chuva. Botucatu-SP, 2009 .

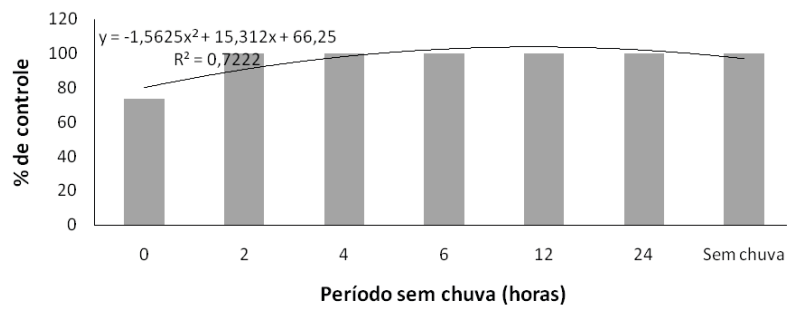

Figura 4 - Porcentagem de controle de plantas de Eichhornia crassipes aos 28 dias após a aplicação do herbicida glyphosate $\left(2.160\right.$ g e.a. ha $\left.{ }^{-1}\right)+0,5 \%$ v/v Aterbane, submetidas a diferentes intervalos sem chuva. Botucatu-SP, 2009 


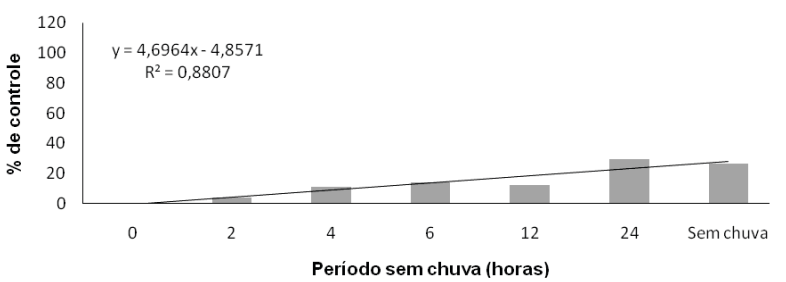

Figura 5 - Porcentagem de controle de plantas de Pistia stratiotes aos 7 dias após a aplicação do herbicida glyphosate $\left(2.160\right.$ g e.a. ha $\left.^{-1}\right)+0,5 \%$ v/v Aterbane, submetidas a diferentes intervalos sem chuva. Botucatu-SP, 2009.

Aos 14 DAA, nota-se que o tratamento que recebeu chuva logo após a aplicação do herbicida apresentou sintomas de injúrias, e todos os demais tratamentos elevaram suas médias de controle, mas não atingiram níveis considerados satisfatórios (Figura 6). Martins et al. (2002), estudando o efeito de glyphosate no controle de $P$. stratiotes sem ocorrência de chuva, com uma dose $150 \%$ maior que a utilizada neste estudo, observaram logo aos 15 DAA 99,5\% de controle, fato que só ocorreu apenas aos 21 DAA neste estudo, o que mostra que doses maiores podem acelerar o processo de controle da espécie, porém não necessariamente significa que menores doses não irão efetivamente controlar as plantas de alface-d'água.

Aos 21 DAA, houve enorme evolução do controle da planta daninha com ocorrência de chuvas a partir de duas horas da aplicação dos herbicidas; todos esses tratamentos apresentaram médias de controle consideradas excelentes, sempre acima de 98\% (Figura 7). Esse fato demonstra que a ocorrência de chuva imediatamente após a aplicação de glyphosate em alface-d'água alterou negativamente o efeito desse herbicida, reduzindo sua eficiência em mais de $81 \%$, quando comparado à inexistência de precipitações.

Na última avaliação realizada neste estudo, aos 28 DAA, foi possivel registrar que todos os períodos de tempo sem chuva após duas horas da aplicação do glyphosate, na formulação Roundup Rodeo, atingiram o máximo de controle da planta daninha, ao passo que, quando da ocorrência de chuva imediatamente após a aplicação do herbicida, o controle foi considerado apenas satisfatório (Figura 8). Foloni \& Pitelli (2005) registraram controle máximo dessa planta daninha sob ação de glyphosate já aos 21 DAA, sem posteriores precipitações, demonstrando assim que o efeito negativo da chuva sobre a ação do glyphosate ocorre apenas com precipitação imediatamente após a aplicação do herbicida e que a ocorrência de chuvas em periodos de tempo de no mínimo duas horas após a aplicação de glyphosate em $P$. stratiotes não afetou sua eficiência de controle desta espécie daninha aquática.

Os resultados obtidos nesses dois estudos mostraram que intervalos maiores que duas horas para a ocorrência de chuva após a aplicação do herbicida glyphosate, em plantas de $E$. crassipes e $P$. stratiotes, são suficientes para

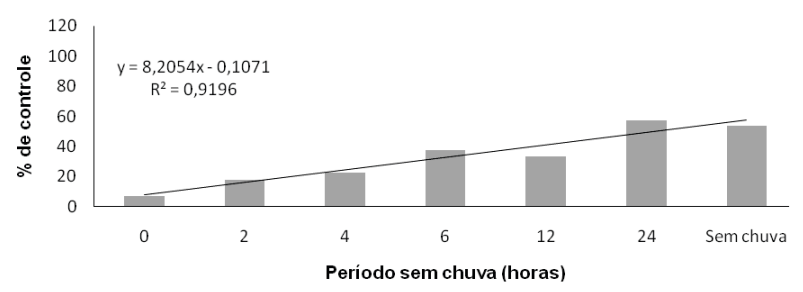

Figura 6 - Porcentagem de controle de plantas de Pistia stratiotes aos 14 dias após a aplicação do herbicida glyphosate $\left(2.160\right.$ g e.a. ha $\left.^{-1}\right)+0,5 \% \mathrm{v} / \mathrm{v}$ Aterbane, submetidas a diferentes intervalos sem chuva. Botucatu-SP, 2009.

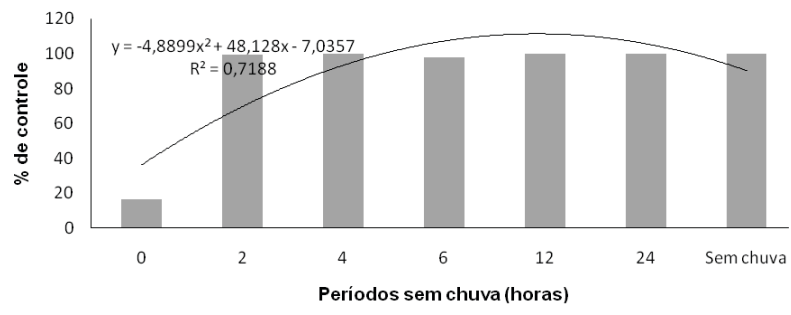

Figura 7 - Porcentagem de controle de plantas de Pistia stratiotes aos 21 dias após a aplicação do herbicida glyphosate $\left(2.160\right.$ g e.a. ha $\left.^{-1}\right)+0,5 \% \mathrm{v} / \mathrm{v}$ Aterbane, submetidas a diferentes intervalos sem chuva. Botucatu-SP, 2009.

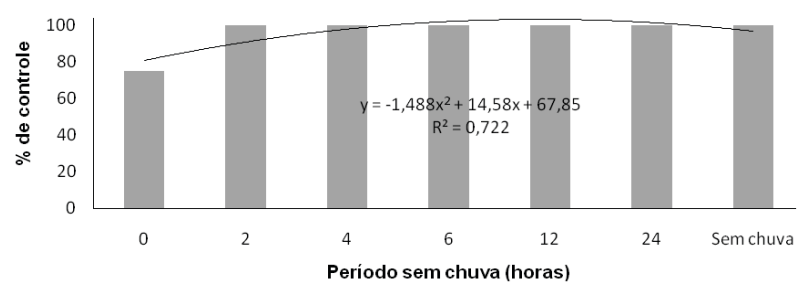

Figura 8 - Porcentagem de controle de plantas de Pistia stratiotes aos 28 dias após a aplicação do herbicida glyphosate $\left(2.160\right.$ g e.a. ha $\left.^{-1}\right)+0,5 \%$ v/v Aterbane, submetidas a diferentes intervalos sem chuva. Botucatu-SP, 2009. 
que todo o potencial desse herbicida seja apresentado, e a inexistência desse intervalo remete a reduções de cerca de $25 \%$ em sua eficiência de controle sobre as duas espécies estudadas.

\section{LITERATURA CITADA}

ANDERSON, M. D.; ARNOLD, W. E. Weed control in sunflowers (Helianthus annuus) with desmediphan and phenmediphan. Weed Sci., v. 32, p. 310-314, 1984.

BRANCO, S. M. Hidrobiologia aplicada à engenharia sanitária. 3.ed. São Paulo: CETESB, 1986. 616 p.

CANCIAN, L. F. et al. Crescimento de Pistia stratiotes em diferentes condições de temperatura e fotoperíodo. Acta Bot. Bras., v. 23, n. 2, p. 552-557, 2009

CÍCERO, E. A. S. et al. Variabilidade genética e sensibilidade de acessos de Pistia stratiotes ao herbicida glyphosate. Planta Daninha, v. 25, n. 3, p. 579-587, 2007.

DANIEL, H. et al. Relationships between macrophytic vegetation and physical features of river habitats: the need for a morphological approach. Hydrobiologia, v. 570, n. 1, p. 11-17, 2006.

DEUBER, R. Controle de plantas daninhas na cultura da soja. In: A soja no Brasil Central. 2.ed. Campinas: Fundação Cargil, 1982. p. 367-392.

FOLONI, L. L.; PITELLI, R. A. Avaliação da sensibilidade de diversas espécies de plantas daninhas aquáticas ao carfentrazone-ethyl, em ambiente controlado.

Planta Daninha, v. 23, n. 2, p. 329-334, 2005.

HOLM, L.; YEO, R. The biology, control and utilization of aquatic weeds. Part I. Weeds Today, v. 81, p. 1-13, 1980.

JAKELAITIS, A. et al. Controle de Digitaria horizontalis pelos herbicidas glyphosate, sulfosate e glifosate potássico submetidos a diferentes intervalos de chuva após a aplicação. Planta Daninha, v. 19, n. 2, p. 279-285, 2001.

KISSMANN, K. G. Plantas infestantes e nocivas. 2.ed. São Bernardo do Campo: BASF, 1997. 852 p.

KRUSE, D. N. et al. Herbicidas inibidores da EPSPS: Revisão de literatura. R. Bras. Herbic., v. 1, n. 2, p. 139-146, 2000.

MARCONDES, D. A. S. et al. Eficiência de fluridone no controle de plantas aquáticas submersas no reservatório de Jupiá. Planta Daninha, v. 21, p. 68-78, 2003. (Edição Especial)

MARTINI, G. et al. Eficácia do herbicida glifosato-potássico submetido à chuva simulada após a aplicação. Bragantia, v. 62, n. 1, p. $39-45,2003$.
MARTINS, D. et al. Controle químico de Pistia stratiotes, Eichhornia crassipes e Salvinia molesta em caixas d'água. Planta Daninha, v. 20, p. 83-88, 2002. (Edição Especial)

MARTINS, D. et al. Caracterização da comunidade de plantas aquáticas de dezoito reservatórios pertencentes a cinco bacias hidrográficas do Estado de São Paulo. Planta Daninha, v. 26, n. 1, p. 17-32, 2008

MARTINS, D. et al. Levantamento da infestação de plantas aquáticas em Porto Primavera antes do enchimento final do reservatório. Planta Daninha, v. 27, p. 879-886, 2009a. (Edição Especial)

MARTINS, D. et al. Ação de adjuvantes na absorção e translocação de glyphosate em plantas de aguapé (Eichhornia crassipes). Planta Daninha, v. 27, n. 1, p. 155-163, 2009b.

MONQUERO, P. A.; SILVA, A. C. Efeito do período de chuva no controle de Euphorbia heterophylla e Ipomoea purpurea pelos herbicidas glyphosate e sulfosate.

Planta Daninha, v. 25, n. 2, p. 399-404, 2007.

NEVES, T. et al. Controle químico do aguapé (Eichhornia crassipes). Planta Daninha, v. 20, p. 89-97, 2002. (Edição Especial)

PEDRINHO JÚNIOR, A. F. F. et al. Momento da chuva após a aplicação e a eficácia dos herbicidas sulfosate e glyphosate aplicados em diferentes formulações. Planta Daninha, v. 20, n. 1, p. 115-123, 2002.

PIRES, N. M. et al. Quantificação dos herbicidas glyphosate e sulfosate na água após simulação de chuva. Planta Daninha, v. 18, n. 3, p. 491-499, 2000

PITELLI, R. L. C. M. et al. Dinâmica da comunidade de macrófitas aquáticas no reservatório de Santana, RJ. Planta Daninha, v. 26, n. 3, p. 473-480, 2008.

SOCIEDADE BRASILEIRA DA CIÊNCIA DAS PLANTAS DANINHAS - SBCPD. Procedimentos para instalação, avaliação e análise de experimentos com herbicidas. Londrina: $1995.42 \mathrm{p}$.

SWAINE, S. M. et al. Forest river plants and water quality in Ghana. Aquatic Bot., v. 85, n. 4, p. 299-308, 2006.

TANAKA, R. H. et al. Ocorrência de plantas aquáticas nos reservatórios da Companhia Energética de São Paulo. Planta Daninha, v. 20, p. 101-111, 2002. (Edição Especial).

VELINI, E. D. et al. Manejo de plantas aquáticas em grandes reservatórios: riscos associados à estratégia de não ação. In: CONGRESSO BRASILEIRO DA CIÊNCIA DAS PLANTAS DANINHAS, 23., 2002, Gramado. Resumos... Gramado: SBCPD, 2002. p. 610

WERLANG, R. C. et al. Efeitos da chuva na eficiência de formulações e doses de glyphosate no controle de Brachiaria decumbens. Planta Daninha, v. 21, n. 1, p. 121-130, 2003. 